\title{
COMPORTAMENTO DE DI-HIDROXIBENZENOS NA DESCOLORAÇÃO DE CROMOTROPE 2R POR REAÇÃO DE FENTON CUPROSA
}

\author{
C. C. V. VELLOSO ${ }^{1}$, C. S. SANTANA ${ }^{1}$ e A. AGUIAR ${ }^{1,2 *}$ \\ ${ }^{1}$ Universidade Federal de São João Del-Rei, Campus Alto Paraopeba, Departamento de \\ Química, Biotecnologia e Engenharia de Bioprocessos \\ ${ }^{2}$ Universidade Federal de Itajubá, Instituto de Recursos Naturais \\ E-mail para contato: camilacaob@hotmail.com
}

\begin{abstract}
RESUMO - A reação de Fenton $\left(\mathrm{Fe}^{2+}+\mathrm{H}_{2} \mathrm{O}_{2} \rightarrow \mathrm{Fe}^{3+}+\cdot \mathrm{OH}+\mathrm{OH}^{-}\right)$é um tipo de processo oxidativo promissor para a degradação de contaminantes orgânicos ao usar radicais $\mathrm{OH}$ a partir de $\mathrm{H}_{2} \mathrm{O}_{2}$. Íons de cobre $\left(\mathrm{Cu}^{+}, \mathrm{Cu}^{2+}\right)$ também podem ser utilizados como catalisadores dessa reação. No presente trabalho foi avaliado o efeito de seis di-hidroxibenzenos (DHBs) que regeneram íons $\mathrm{Cu}^{+}$para aumentar a descoloração do corante cromotrope $2 \mathrm{R}$ por reação de Fenton cuprosa. Reações com $\mathrm{Cu}^{2+}$ promoveram índices mais altos de descoloração e o ácido 2,5-dihidroxibenzoico foi o único DHB que aumentou a descoloração, de $56 \%$ para apenas $60 \%$ após 60 min de reação. Para o $\mathrm{Cu}^{+}$, todos os DHBs diminuíram a descoloração do corante em estudo. Os DHBs avaliados apresentaram predominantemente propriedades antioxidantes, ao contrário de estudos anteriores do próprio grupo de pesquisa que observaram efeitos pro-oxidantes em reações similares de descoloração usando íons de ferro.
\end{abstract}

\section{INTRODUÇÃO}

A indústria têxtil é fonte geradora de grandes quantidades de efluentes contendo forte coloração e elevada carga orgânica provenientes do processo de tingimento. A maior parte da carga orgânica é representada por agentes engomantes (amido), facilmente degradados pelos processos biológicos tradicionais. No entanto, a cor, originada por uma grande variedade de corantes reativos, tipo azo com elevado potencial carcinogênico e mutagênico, mostra-se bastante resistente a este tipo de degradação (Braga et al., 2002). Pelo fato das técnicas tradicionais de remediação utilizadas pela indústria serem ineficientes para a remoção da cor e toxicidade, há necessidade em desenvolver novas alternativas de tratamento. Dentre elas, os processos oxidativos avançados (POAs) merecem destaque pela elevada capacidade de degradação em tempos relativamente curtos (Aguiar et al., 2007).

Os POAs são baseados na formação de radicais livres, principalmente o radical $\mathrm{OH}$ hidroxila $\left({ }^{\bullet} \mathrm{OH}\right)$, agente altamente oxidante. Devido a sua elevada reatividade $\left(\mathrm{E}^{\mathrm{o}}=2,81 \mathrm{~V}\right)$, o mesmo pode reagir com uma grande variedade de compostos, podendo promover a total mineralização de poluentes orgânicos a $\mathrm{CO}_{2}$ e $\mathrm{H}_{2} \mathrm{O}$ ou, no caso de pré-tratamentos, tornandoos biodegradáveis. Tais processos são divididos em sistemas homogêneos e heterogêneos, nos quais os radicais hidroxila podem ser gerados com ou sem irradiação ultravioleta (Oppenlander, 2003). 
Um dos POA mais promissores consiste na reação entre $\mathrm{Fe}^{2+}$ e $\mathrm{H}_{2} \mathrm{O}_{2}$, conhecida como reação de Fenton $\left(\mathrm{Fe}^{2+}+\mathrm{H}_{2} \mathrm{O}_{2} \rightarrow \mathrm{Fe}^{3+}+{ }^{\bullet} \mathrm{OH}+\mathrm{OH}^{-}\right)$. O cobre atua como um catalisador na decomposição de $\mathrm{H}_{2} \mathrm{O}_{2}$ (reação de Fenton cuprosa), de modo similar ao ferro. Ambos metais de transição reagem com $\mathrm{H}_{2} \mathrm{O}_{2}$ formando complexos intermediários que em seguida se decompõem formando o radical $\mathrm{OH}$. Na reação de Fenton cuprosa, o complexo formado entre $\mathrm{H}_{2} \mathrm{O}_{2}$ e o metal é mais estável que na reação de Fenton ferrosa. Como consequência, as espécies ativadas da reação de Fenton cuprosa $\left(\mathrm{Cu}^{+}+\mathrm{H}_{2} \mathrm{O}_{2} \rightarrow \mathrm{Cu}^{2+}+{ }^{\bullet} \mathrm{OH}+\mathrm{OH}^{-}\right)$podem ser o radical $\mathrm{OH}$ ou ainda o íon $\mathrm{Cu}^{3+}$. A reação de Fenton cuprosa é aproximadamente 3 vezes mais rápida que a ferrosa, no entanto, a estabilidade do $\mathrm{Cu}^{+}$em solução aquosa depende da presença de quelantes ou compostos que reduzam constantemente $\mathrm{Cu}^{2+} \mathrm{a} \mathrm{Cu}^{+}$(Aguiar et al., 2007; Nichela et al., 2013).

A fim de aumentar a produção de radicais livres por reagentes Fenton, no presente trabalho, avaliou-se o efeito de cinco di-hidroxibenzenos (DHBs): catecol e ácidos 2,3-dihidroxibenzoico, 2,4-di-hidroxibenzoico, 2,5-di-hidroxibenzoico e 3,4-di-hidroxifenilacético, além do tri-hidroxibenzeno ácido gálico. Esses fenóis reduzem íons $\mathrm{Cu}^{2+} \mathrm{a} \mathrm{Cu}^{+}$e foram avaliados no presente trabalho como mediadores para aumentar a descoloração do azocorante cromotrope2R (Figura 1) por reação de Fenton cuprosa. As estruturas químicas dos DHBs e do ácido gálico estudados no presente trabalho encontram-se ilustradas na Figura 2.<smiles>O=S(=O)(O)c1cc2cc(S(=O)(=O)O[Na])cc(O)c2c(O)c1N=Nc1ccccc1</smiles>

Figura 1 - Estrutura química do azocorante cromotrope 2R. 
<smiles>O=C(O)c1cc(O)ccc1O</smiles>

(a)<smiles>O=C(O)c1cc(O)c(O)c(O)c1</smiles>

(d)<smiles>Oc1ccccc1O</smiles>

(b)<smiles>O=C(O)c1ccc(O)cc1O</smiles>

(e)<smiles>O=C(O)c1cccc(O)c1O</smiles>

(c)<smiles>O=C(O)Cc1ccc(O)c(O)c1</smiles>

(f)

Figura 2: Estrutura química dos compostos fenólicos redutores de cobre estudados no trabalho em questão: (a) ácido 2,5-di-hidroxibenzoico; (b) catecol; (c) ácido 2,3-di-hidroxibenzoico; (d) ácido gálico; (e) ácido 2,4-di-hidroxibenzoico; (f) ácido 3,4-di-hidroxifenilacético.

\section{MATERIAIS E MÉTODOS}

Os experimentos de descoloração foram conduzidos em cubetas de quartzo de $3 \mathrm{~mL}$ contendo $200 \mu \mathrm{L}$ de $\mathrm{H}_{2} \mathrm{O}_{2} 4,5 \mathrm{mmol} \mathrm{L}^{-1}, 200 \mu \mathrm{L}$ de cromotrope $2 \mathrm{R} 300 \mu \mathrm{mol} \mathrm{L} \mathrm{L}^{-1}, 200 \mu \mathrm{L}$ de $\mathrm{CuCl} 300 \mu \mathrm{mol} \mathrm{L}{ }^{-1}$ ou $200 \mu \mathrm{L}$ de $\mathrm{CuCl}_{2} 300 \mu \mathrm{mol} \mathrm{L}{ }^{-1}$. Quando realizados os experimentos na presença dos DHBs ou ácido gálico, $200 \mu \mathrm{L}$ de solução $100 \mu \mathrm{mol} \mathrm{L}^{-1}$ de mediadores foram adicionados. Avolumou-se a cubeta para $2 \mathrm{~mL}$, sendo mantida ao abrigo da luz. Após tempos fixos de reação, a cubeta contendo amostra foi inserida no espectrofotômetro e realizaram-se as medidas de absorbância no comprimento de onda referente à máxima absorção do corante em estudo $(\lambda=512 \mathrm{~nm})$. A degradação do corante foi monitorada por meio da descoloração das soluções em intervalos de 5, 10, 20, 40 e 60 minutos de reação. Preparou-se o branco com água deionizada. Os ensaios foram realizados em duplicata e à temperatura ambiente. $\mathrm{O}_{2} \mathrm{O}_{2}$ residual foi quantificado com $\mathrm{NH}_{4} \mathrm{VO}_{3}$ em $450 \mathrm{~nm}$ (Aguiar e Ferraz, 2007). As reações de descoloração na ausência dos mediadores foram também realizadas bem como aquelas na ausência de corantes. Por meio destas últimas objetivou-se investigar a possível interferência dos produtos de degradação dos DHBs ou ácido gálico na descoloração dos corantes. 


\section{RESULTADOS E DISCUSSÕES}

Por meio das reações realizadas na ausência do corante cromotrope $2 \mathrm{R}$, foi possível observar que os produtos de degradação dos DHBs não ocasionaram o surgimento de coloração no meio reacional, ou seja, não interferiram no processo de descoloração do composto-alvo. Estudos anteriores alegam que a oxidação dos azocorantes, classificação em que se enquadra o cromotrope $2 \mathrm{R}$, procede pela adição de um radical hidroxila ao átomo de carbono que suporta a ligação $-\mathrm{N}=\mathrm{N}-$ (Lachheb et al., 2002).

A descoloração do cromotrope $2 \mathrm{R}$ por $\mathrm{Cu}^{+} / \mathrm{H}_{2} \mathrm{O}_{2}$ e $\mathrm{Cu}^{+} / \mathrm{H}_{2} \mathrm{O}_{2}$ está mostrada nas Figuras 3 e 4, respectivamente. Para o comportamento cinético é notável que em todas as reações, a descoloração do cromotrope $2 \mathrm{R}$ foi mais rápida nos 5 primeiros minutos de reação e posteriormente permaneceu inalterada. Estudos anteriores alegam que a descoloração de corantes por radicais $\mathrm{OH}$ pode ser dividida em dois estágios. O primeiro estágio corresponde à formação e degradação por radicais hidroxila, que ocorre rapidamente e, portanto, a descoloração é mais intensa. O segundo refere-se àquele no qual, devido à menor formação de radicais hidroxila e início da formação de radicas hidroperoxila $\left(\mathrm{HO}_{2} \bullet\right)$, tem-se uma descoloração mais lenta (Du et al., 2006). Esse segundo estágio também pode ser atribuído ao balanço entre surgimento e desaparecimento de radicais $\mathrm{OH}$.

O efeito de DHBs e ácido gálico foi avaliado para as reações contendo tanto íons $\mathrm{Cu}^{+}$ (Figura 3) quanto $\mathrm{Cu}^{2+}$ (Figura 4). De acordo com a Figura 3, notou-se que todos os DHB e o ácido gálico diminuíram a descoloração do cromotrope $2 \mathrm{R}$. O catecol e o ácido gálico foram os fenóis que apresentaram maior inibição, enquanto os ácidos 2,4- e 2,5-di-hidroxibenzoico foram os que apresentaram menor inibição. Essa propriedade antioxidante pode estar relacionada ao sequestro de radicais $\mathrm{OH}$ por esses mediadores, por meio da doação de prótons (Briante et al., 2003).

$\mathrm{Na}$ Figura 4, observou-se que o ácido 2,5-di-hidroxibenzoico aumentou a descoloração do corante por $\mathrm{Cu}^{2+} / \mathrm{H}_{2} \mathrm{O}_{2}$, de $56 \%$ para discretos $60 \%$ de descoloração enquanto o ácido 2,4di-hidroxibenzoico não demonstrou efeito algum. Por outro lado, os outros DHBs e o ácido gálico apresentaram inibição na descoloração. 


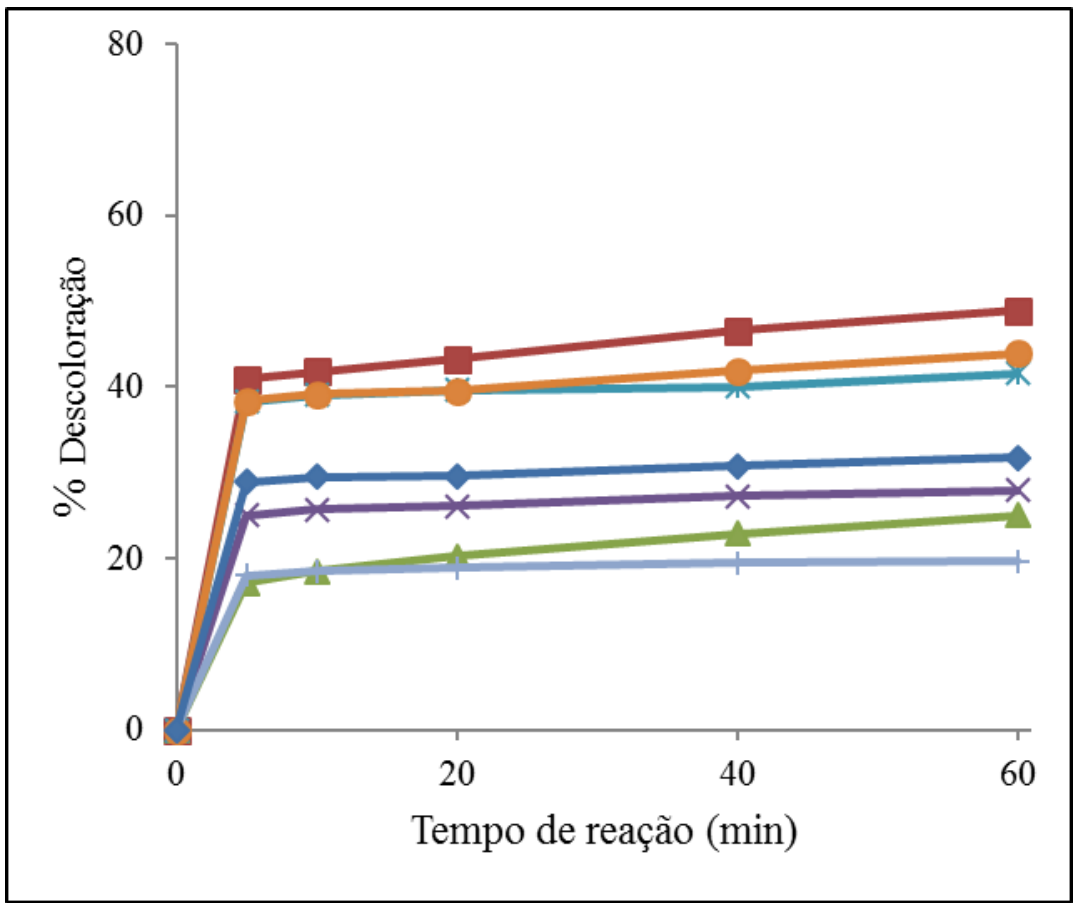

Figura 3 - Curvas de descoloração do corante cromotrope $2 \mathrm{R} 30 \mu \mathrm{mol} \mathrm{L} \mathrm{L}^{-1}$, promovidas por: $30 \mu \mathrm{mol}$ $\mathrm{L}^{-1}$ de $\mathrm{Cu}^{+}, 450 \mu \mathrm{mol} \mathrm{L} \mathrm{L}^{-1}$ de $\mathrm{H}_{2} \mathrm{O}_{2}$ e $10 \mu \mathrm{mol} \mathrm{L}^{-1}$ de composto fenólico. Ausência de mediador fenólico $(--)$; ácido gálico $(-\infty)$; ácido 2,3-di-hidroxibenzoico $(\leftarrow)$; ácido 2,4-di-hidroxibenzoico $(-$ *); ácido 2,5-di-hidroxibenzoico ( --$)$; catecol $(-)$ e ácido 3,4-di-hidroxifenilacético $(-\bullet)$. Os experimentos de descoloração foram realizados em duplicata e apresentaram erros inferiores a 5\% quando comparados ao valor médio. 


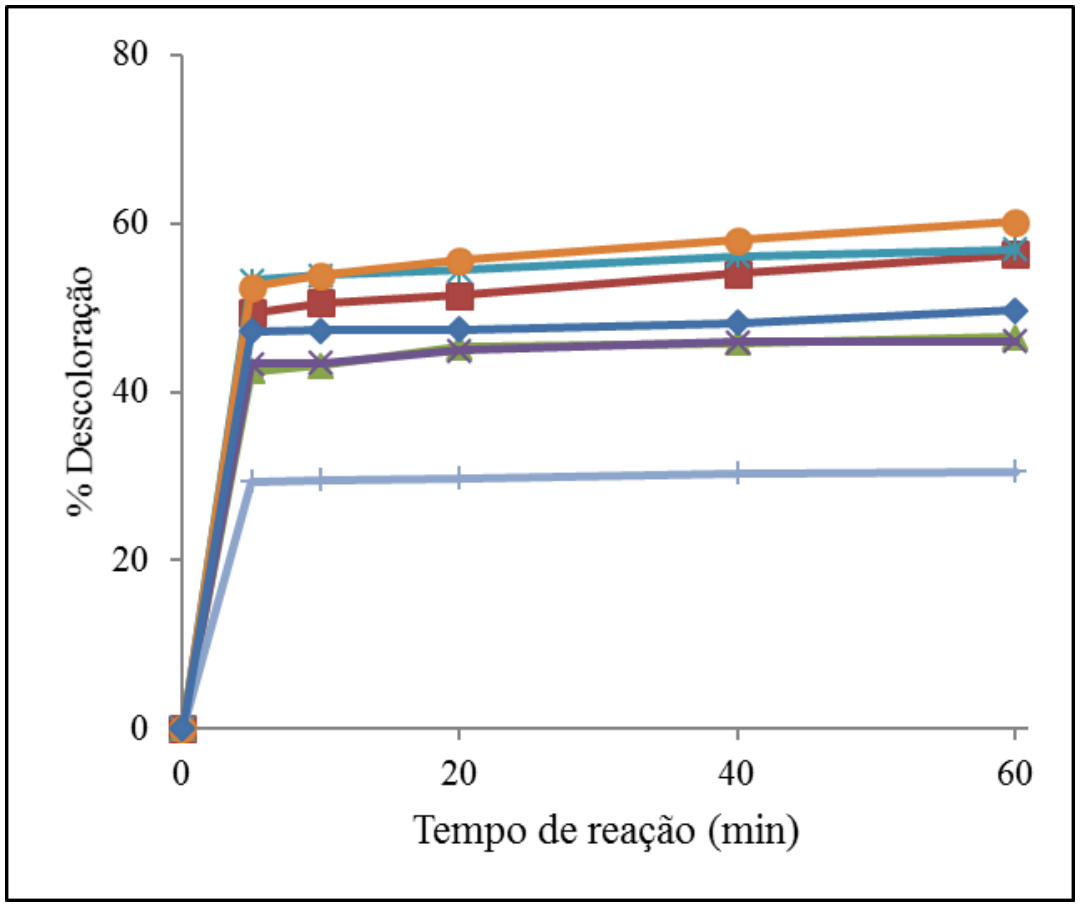

Figura 4 - Curvas de descoloração do corante cromotrope $2 \mathrm{R} 30 \mu \mathrm{mol} \mathrm{L}^{-1}$, promovidas por: $\quad 30$ $\mu \mathrm{mol} \mathrm{L}{ }^{-1}$ de $\mathrm{Cu}^{2+}, 450 \mu \mathrm{mol} \mathrm{L}^{-1}$ de $\mathrm{H}_{2} \mathrm{O}_{2}$ e $10 \mu \mathrm{mol} \mathrm{L}^{-1}$ de composto fenólico. Ausência de mediador fenólico $(-\leftarrow)$; Ácido gálico $(-\rightarrow)$; ácido 2,3-di-hidroxibenzoico $(\multimap)$; ácido 2,4-dihidroxibenzoico ( $*$ ); ácido 2,5-di-hidroxibenzoico $(--)$; catecol $(-$ ) e ácido 3,4-dihidroxifenilacético $(\neg \bullet)$. Os experimentos de descoloração foram realizados em duplicata e apresentaram erros inferiores a 5\% quando comparados ao valor médio.

Além de diminuir a descoloração (efeito antioxidante), em todas as reações com $\mathrm{Cu}^{+}$os DHBs e o ácido gálico promoveram um pequeno aumento no consumo de $\mathrm{H}_{2} \mathrm{O}_{2}$, como é apresentado na Tabela 1. Por outro lado, para as reações com $\mathrm{Cu}^{2+}$ os mediadores fenólicos diminuíram o consumo de $\mathrm{H}_{2} \mathrm{O}_{2}$. Vale destacar o ácido 2,5-dihidroxibenzoico, o qual aumentou um pouco a descoloração do corante requerendo um menor consumo de $\mathrm{H}_{2} \mathrm{O}_{2}$ em relação à reação não mediada. 
Tabela $1-\mathrm{H}_{2} \mathrm{O}_{2}$ consumido (\%) após 60 minutos de reação para descoloração do cromotrope $2 \mathrm{R}$ por reagentes Fenton $\left(\mathrm{Cu}^{+} / \mathrm{H}_{2} \mathrm{O}_{2} \mathrm{e} \mathrm{Cu}^{2+} / \mathrm{H}_{2} \mathrm{O}_{2}\right)$ na presença ou ausência de DHBs

\begin{tabular}{|c|c|c|c|c|}
\hline \multirow{2}{*}{ Sistema Reacional } & \multicolumn{4}{|c|}{$\%$ de $\mathrm{H}_{2} \mathrm{O}_{2}$ Consumido } \\
\cline { 2 - 5 } & \multicolumn{2}{|c|}{ Presença do corante } & \multicolumn{2}{c|}{ Ausência do corante } \\
\cline { 2 - 5 } $\mathrm{Cu}^{+} / \mathrm{H}_{2} \mathrm{O}_{2}$ & $\mathrm{Cu}^{2+} / \mathrm{H}_{2} \mathrm{O}_{2}$ & $\mathrm{Cu}^{+} / \mathrm{H}_{2} \mathrm{O}_{2}$ & $\mathrm{Cu}^{2+} / \mathrm{H}_{2} \mathrm{O}_{2}$ \\
\hline Ausência de DHBs & $55,2 \pm 0$ & $65,6 \pm 0,65$ & - & - \\
\hline $\begin{array}{c}\text { Ácido 2,3-di- } \\
\text { hidroxibenzoico }\end{array}$ & $58,3 \pm 0,6$ & $54,4 \pm 0,3$ & $54,4 \pm 0,3$ & $54,4 \pm 0,7$ \\
\hline $\begin{array}{c}\text { Ácido 2,4-di- } \\
\text { hidroxibenzoico }\end{array}$ & $55,5 \pm 0,3$ & $57,4 \pm 0,3$ & $61,7 \pm 0$ & $60,0 \pm 1,7$ \\
\hline $\begin{array}{c}\text { Ácido 2,5-di- } \\
\text { hidroxibenzoico }\end{array}$ & $60,0 \pm 0,3$ & $53,9 \pm 1,3$ & $64,3 \pm 0,7$ & $63,6 \pm 0$ \\
\hline $\begin{array}{c}\text { Ácido gálico } \\
\text { Ácido 3,4-di- } \\
\text { hidroxifenilacético }\end{array}$ & $57,7 \pm 0,6$ & $57,4 \pm 0,3$ & $69,3 \pm 0,6$ & $68,3 \pm 0,3$ \\
\hline catecol & $61,7 \pm 0,7$ & $63,9 \pm 0,3$ & $64,0 \pm 1,0$ & $63,3 \pm 1,0$ \\
\hline
\end{tabular}

Como o consumo de $\mathrm{H}_{2} \mathrm{O}_{2}$ foi significativo nas reações, acima de $50 \%$, pode-se inferir que os mediadores fenólicos aparentemente não evitaram a reação do cobre com $\mathrm{H}_{2} \mathrm{O}_{2}$, mas de fato eles atuaram como sequestradores de radicais $\mathrm{OH}$. Esses radicais também podem reagir com seu próprio precursor ao invés de atuarem no processo de descoloração do corante. Nesse segundo caso, tem-se a geração de um novo radical, o hidroperoxila $\left({ }^{\bullet} \mathrm{OH}+\mathrm{H}_{2} \mathrm{O}_{2} \rightarrow \mathrm{H}_{2} \mathrm{O}+\right.$ $\mathrm{HO}_{2}{ }^{\bullet}$ ), o qual possui menor potencial padrão de redução que o radical $\mathrm{OH}$.

\section{CONCLUSÕES}

Os DHB avaliados apresentaram predominantemente propriedades antioxidantes, ao contrário de estudos anteriores que observaram efeitos pro-oxidantes desses compostos em reações similares de descoloração usando íons $\mathrm{Fe}^{2+}$ ou $\mathrm{Fe}^{3+}$. Apenas o sistema $\mathrm{Cu}^{2+} / \mathrm{H}_{2} \mathrm{O}_{2} / 2,5-$ di-hidroxibenzoico aumentou o índice de descoloração do cromotrope $2 \mathrm{R}$ requerendo para isso um menor consumo de $\mathrm{H}_{2} \mathrm{O}_{2}$. Na presença de $\mathrm{Cu}^{+}$e mediador fenólico, houve um maior consumo de peróxido, ao contrário do que aconteceu quando foi utilizado o $\mathrm{Cu}^{2+}$.

\section{AGRADECIMENTOS}

Os autores agradecem à FAPEMIG (processo APQ-01585-11) e à concessão de bolsa de iniciação científica PIBIC/CNPq. 


\section{REFERÊNCIAS}

ABDELMALEK, F.; GHEZZAR, M. R.; BELHADJ, M.; ADDOU, A.; BRISSET J. L. Bleaching and degradation of textile dyes by nonthermal plasma process at atmospheric pressure. Ind. Eng. Chem. Research, v. 45, p. 23-29, 2006.

AGUIAR, A.; FERRAZ, A.; CONTRERAS, D.; RODRÍGUEZ, J. Mecanismo e aplicações da reação de Fenton assistida por compostos fenólicos. Quim. Nova, v. 30, p. 623-628, 2007.

AGUIAR, A.; FERRAZ, A. Fe3+- and Cu2+-reduction by phenol derivatives associated with Azure B degradation in Fenton-like reactions. Chemosphere, v. 66, p. 947-954, 2007.

BRAGA, B.; HESPANHOL, I.; CONEJO, J. G. L.; BARROS, M. T. L.; SPENCER, M.; PORTO, M.; NUCCI, N.; JULIANO, N.; EIGER, S. Introdução à Engenharia Ambiental. São Paulo: Prentice Hall, 2002.

BRIANTE, R.; FEBBRAIO, F.; NUCCI, R. Antioxidant properties of low molecular weight phenols present in the Mediterranean diet. J. Agric. Food Chem., v. 51, p. 6975-6981, 2003.

DU, Y.; ZHOU M.; LEI L. Role of the intermediates in the degradation of phenolic compounds by Fenton-like process. J. Hazard. Mat., v. 136, p. 859-865, 2006.

LACHHEB, H.; PUZENAT, E.; HOUAS, A.; KSIBI, M.; ELALOUI, E.; GUILLARD, C.; HERRMANN, J. M. Photocatalytic degradation of various types of dyes (Alizarin S, Crocein Orange G, Methyl Red, Congo Red, Methylene Blue) in water by UVirradiated titania. App. Catal. B: Environ., v. 39, p. 75-90, 2002.

MAGUREANU, M.; MANDACHE, N. B.; PARVULESCU, V. I. Degradation of organic dyes in water by electrical discharges. Plasma Chem. Plasma Process., v. 27, p. 589598, 2007.

NICHELA A. D.; BERKOVIC M. A.; COSTANTE R. M.; JULIARENA P. M.; EINSCHLAG G. S. F. Nitrobenzene degradation in Fenton-like systems using $\mathrm{Cu}(\mathrm{II})$ as catalyst.Comparison between $\mathrm{Cu}(\mathrm{II})$ - and Fe(III)-based systems. Chem. Eng. J., v. 228, p. 1148-1157, 2013.

OPPENLANDER, T. Photochemical Purification of Water and Air Treatment. Weinheim (Cambridge): Willey-VCH, 2003. 